\title{
Real-Time Trading Strategies of Proactive DISCO with Heterogeneous DG Owners.
}

Zhang, Chunyu; Wang, Qi; Wang, Jianhui; Pinson, Pierre; Østergaard, Jacob

Published in:

IEEE Transactions on Smart Grid

Link to article, DOI:

10.1109/TSG.2016.2597263

Publication date:

2017

Document Version

Peer reviewed version

Link back to DTU Orbit

Citation (APA):

Zhang, C., Wang, Q., Wang, J., Pinson, P., \& Østergaard, J. (2017). Real-Time Trading Strategies of Proactive DISCO with Heterogeneous DG Owners. IEEE Transactions on Smart Grid, 9(3), 1688 - 1697.

https://doi.org/10.1109/TSG.2016.2597263

\section{General rights}

Copyright and moral rights for the publications made accessible in the public portal are retained by the authors and/or other copyright owners and it is a condition of accessing publications that users recognise and abide by the legal requirements associated with these rights.

- Users may download and print one copy of any publication from the public portal for the purpose of private study or research.

- You may not further distribute the material or use it for any profit-making activity or commercial gain

- You may freely distribute the URL identifying the publication in the public portal 


\title{
Real-Time Trading Strategies of Proactive DISCO with Heterogeneous DG Owners
}

\author{
Chunyu Zhang, Member, IEEE, Qi Wang, Member, IEEE, Jianhui Wang, Senior Member, IEEE, \\ Pierre Pinson, Senior Member, IEEE, and Jacob Østergaard, Senior Member, IEEE
}

\begin{abstract}
This paper presents a methodology to obtain the optimal trading strategies between the proactive distribution company (PDISCO), heterogeneous distributed generation owners (DGOs) and wholesale market in a real-time trading framework. In this framework, the PDISCO's decisions cover the power procurements from DGOs and the transactions within the real-time market. A one-leader multi-follower-type bilevel model is proposed to embody the PDISCO-DGO gaming structure. The upper-level (UL) problem is to maximize the PDISCO's profit, while the lower-level (LL) problem indicates the profit maximization per DGO. Since the UL problem is non-linear and non-convex and the $L L$ problems are linear and convex, we reformulate the proposed model to a solvable mathematical program with equilibrium constraints (MPEC) by an equivalent primal-dual approach. The numerical results of the case studies show the effectiveness and scalability of the proposed model.
\end{abstract}

Index Terms-Distributed generation (DG), proactive distribution company (PDISCO), distributed generation owner (DGO), Bayesian game, bilevel model, multi-period AC power flow, mathematical program with equilibrium constraints (MPEC).

\section{NOMENCLATURE}

Sets and Indices

$i, j, B$
$i j, \Lambda$
$d, D$
$g, G$
$s, S$
$m, M$
$n, N$
$k, K$
$t, T$

$\omega, \Omega$

Index and set of distribution buses.

Index and set of distribution feeders.

Index and set of demands.

Index and set of stochastic DGs.

Index and set of storage devices (SDs).

Index and set of DGOs with stochastic DGs. Index and set of DGOs with only SDs.

Index and set of DGOs with both stochastic DGs and SDs.

Index and set of time periods (e.g., hours per day).

Index and set of scenarios.

The work of C. Zhang and Q. Wang was supported in part by the project 255209 from the Research Council of Norway, and in part by the Danish iPower platform project 10-095378. J. Wang's work was supported by the U.S. Department of Energy (DOE)'s Office of Electricity Delivery and Energy Reliability.

C. Zhang is with the Department of Electric Power Engineering, Norwegian University of Science and Technology, 7034, Trondheim, Norway (e-mail: chunyu.zhang@ntnu.no).

Q. Wang is with Nordea, Filial of Nordea Bank AB, (Publ.), 1401, Copenhagen K, Denmark (e-mail: Qi.Wang@ nordea.com).

J. Wang is with the Energy Systems Division, Argonne National Laboratory, Argonne, IL 60439, USA (e-mail: jianhui.wang@anl.gov).

P. Pinson and J. Østergaard are with the Center for Electric Power and Energy, Technical University of Denmark, 2800, Kgs. Lyngby, Denmark (email: ppin@elektro.dtu.dk; joe@elektro.dtu.dk).

Manuscript received

$\mathcal{M}_{D} \quad$ Mapping of the set of demands onto the set of buses.

$\mathcal{M}_{G}, \mathcal{M}_{S} \quad$ Mapping of the set of DGs or SDs onto the set of buses respectively.

$\mathcal{M}_{O} \quad$ Mapping of the set of DGs and SDs onto the set of DGOs.

\section{Variables $\lambda_{t(m, k, n)}^{B(1,2,3)}$ $\lambda_{t(m, k, n)}$ $P_{t(m, k, n)}^{B(1,2,3)}$ \\ $P^{T P(1,2,3)}$ $P_{t(m, k, n) \omega}$ $Q_{t(m, k, n) \omega}^{T P(1,2,3)}$}

$P_{t g \omega}^{d e v}, Q_{t g \omega}^{d e v}$

$P_{t s \omega}^{s d}, Q_{t s \omega}^{s d}$

$P_{t s \omega}^{s c}, Q_{t s \omega}^{s c}$

$E_{t s \omega}$

$P_{t \omega}^{R T}, Q_{t \omega}^{R T}$

$P_{t d \omega}^{s h e d}, Q_{t d \omega}^{\text {shed }}$

$Q_{t i \omega}^{C}$

$P_{t, i j, \omega}^{f}, Q_{t, i j, \omega}^{f}$

$e_{t i \omega}, \delta_{t i \omega}$

Parameters

$P_{t g \omega}^{D G}, Q_{t g \omega}^{D G}$

$C_{g}$

$C_{s}^{s d}, C_{s}^{s c}$

$\bar{P}_{s}^{D G} \frac{C_{Q}}{Q} D G$

$\bar{P}_{s}^{s d}, \bar{Q}_{s}^{s d}$

$\bar{P}_{s}^{s c}, \bar{Q}_{s}^{s c}$
Bidding price and generation quantity of respective Type1,2,3 DGOs at time $t$.

Active and reactive power generated by respective Type1,2,3 DGOs at time $t$ for scenario $\omega$.

Active and reactive power bought from the PDISCO for DG $g$ by Type1 DGOs at time $t$ for scenario $\omega$.

Active and reactive power discharged by SD $s$ at time $t$ for scenario $\omega$.

Active and reactive power charged by SD $s$ at time $t$ for scenario $\omega$.

Residual energy of SD $s$ at time interval $t$ for scenario $\omega$.

Active and reactive power exchanging in realtime market at time $t$ for scenario $\omega$.

Active and reactive power of load-shedding at demand $d$ at time $t$ for scenario $\omega$.

Reactive power supplied by the shunt compensator at bus $i$ at time $t$ for scenario $\omega$.

Active and reactive power flows through feeder $i j$ at time $t$ for scenario $\omega$.

Voltage magnitude and phase angle at bus $i$ at time $t$ for scenario $\omega$.

Active and reactive power generation realization of DG $g$ at time $t$ for scenario $\omega$.

Generating cost of DG $g$.

Discharging and charging cost of SD $s$.

Maximum active and reactive power generation bounds of stochastic DG $g$.

Maximum active and reactive power discharging bounds of SD $s$.

Maximum active and reactive power charging bounds of SD $s$. 
$\underline{E}_{s}, \bar{E}_{s} \quad$ Lower and upper bounds of residual energy of SD $s$.

$\eta_{s}^{s d}, \eta_{s}^{s c} \quad$ Discharging and charging energy efficiencies of SD $s$.

$P_{t}^{D A}, Q_{t}^{D A} \quad$ Active and reactive power purchased from the day-ahead market at time $t$.

$P_{t d}^{D}, Q_{t d}^{D}$

$\lambda_{t}^{D A}$

$\lambda_{t}^{R T}$

$\lambda_{t}^{D}$

$\lambda_{t}^{\text {shed }}$

$\lambda_{t}^{\text {pen }}$

$\lambda_{t}^{s c}$

$\bar{S}, \bar{S}_{i j}$

$\underline{Q}_{i}^{C}, \bar{Q}_{i}^{C}$

$\underline{e}_{i}, \bar{e}_{i}$

$\tau_{i}$

$G_{i j}, B_{i j}, b_{i j}$ Conductance, susceptance and charging susceptance of feeder $i j$.

$\Upsilon_{t(m, k, n)}^{T P(1,2,3)} \quad$ Profit guarantee factor of the DGO of Type1,2,3 respectively at time $t$.

\section{INTRODUCTION}

$\mathbf{H}$ IGH integration of DGs in the distribution network inspires a possible deregulated environment for the distribution company (DISCO) directly procuring the DGs' productions in the local area. In the U.S., one of the latest initiatives is to make regulatory changes to establish a distribution-level market for cost-effective utilization of DGs, as addressed in the New York Reforming Energy Vision (NY REV) [1]. Such an advisable insight can motivate the distributed generation owner (DGO) to play an essential role as an emerging business entity to trade with the DISCO. To this end, the DISCO has to make optimal decisions about the wholesale market transactions and the local power procurements.

Traditionally, the DISCO purchases electricity from the wholesale market and supplies the demands in a singledirection. Currently, besides supplying the local demands, the advanced smart grid technology enables the DISCO to perform in a bi-direction power exchanging fashion, i.e. selling or purchasing electricity with the transmission-level markets. This provides an ambitious scheme for the DISCO to sell the excess power (procured from DGOs) to the transmissionlevel markets profitably, acting as an active electricity producer. With the characteristics above, the proactive DISCO (PDISCO) is so-defined in this paper.

As reviewed in [2], since the distribution-level DGs are small-scale, diverse, and dispersed, the differing ownerships of DGs can render heterogeneous DGOs. As profit-driven entities, the DGOs with stochastic DGs have to consider their production uncertainties, while the DGOs with SDs have to consider their price-based discharging/charging. In addition, with the superiority of DGs' quick response and low cost, the DGOs are more conducive to participate in the real-time trading process. Thus, we propose a real-time trading framework to capture a PDISCO's trading strategies interacting with various types of DGOs.

In order to obtain the real-time trading strategies, the PDISCO trading with heterogeneous DGOs can be formulated as a one-leader multi-follower-type Bayesian game model [3], following a bilevel structure. At each time $t$ : Categorized in a certain follower type, a lower-level (LL) problem per DGO is to maximize its own profit (minimizing minusprofit) by behaving rationally on bidding prices and generation quantities, in which the scenario-based uncertainty handling method [4] can be adopted to characterize the DGs' stochastic productions. The upper-level (UL) problem represents the minus-profit minimization of the PDISCO with the optimal decisions on offering prices and power procurements.

Note that the UL problem is non-linear and non-convex, while the LL problems are linear and thus convex. The proposed bilevel model is complex, but solvable by reformulating it to an MPEC with an alternative approach, such as a primaldual approach [5].

In the literature, to achieve a competitive circumstance, a static day-head DISCO acquisition market model is proposed in [6], including a Pool setup and bilateral contracts. The energy providers are seen as the wholesale market, independent/self-owned DGs, and load curtailment options. However, the DGs are assumed to be dispatchable, which is not realistic since the DGs' outputs are intermittent and uncontrollable. In [7], to address the competition between the participants, a DISCO-ISO bilevel model is presented to deal with the DGs and interruptible loads in a day-ahead market. The UL problem seeks revenue maximization of the DISCO, and the LL simulates the ISO's market clearing problem. The DGs are only considered as DISCO self-owned units, which limits the liberty of the DGs' ownership. Additionally, the DGs' output and load-shedding price are fixed, while the network constraints are not accounted for. Taking the DGO's interests into account, the DGO-DISCO contract pricing is also issued by a bilevel model in [8]. The UL problem is to maximize one DGO's profit equipped with the network constraints listed in the LL problem, but the formulation is simplified by only concerning the active power and voltage value. At the distribution level, a market framework is presented in [9], interacting with the transmission-level markets, the PDISCO's procurement strategies between the distributionlevel aggregator-based demand responses (DRs) and wholesale market transactions are derived through a proposed one-leader multi-follower bilevel model [10]. As fully discussed in [11][14], the bilevel modeling has been widely used to identify the wholesale market outcomes with multiple game players during the decision-making process.

To achieve the cost-effective use of distributed energy resources (DERs), a cake cutting game is investegated in [15] to price energy in a smart community. In [16], a hybrid 
stochastic/robust optimization model is proposed to render a bidding strategy for a microgrid (MG) to participate in the dayahead market. At the real-time stage, a multiagent-based gametheoretic reverse auction model is presented in [17] for $\mathrm{MG}$ market operations. Considering the uncertainties of market prices, the loss payment is minimized in [18] by scheduling DR and energy storage system (ESS). To maximize the profit of a commercial virtual power plant (CVPP), a three-stage stochastic bilevel model is used to make offering strategies on the basis of various DERs and storages [19]. From the management perspective, a dual-horizon rolling scheduling framework is presented in [20] to optimally operate a set of DERs. An hourly electricity pricing scheme is reported in [21] to build network tariffs for mobilizing price-responsive customers. To exploit DG's benefits, a multi-agent systembased modeling of an active distribution network is studied in [22] to enhance control strategies for decreasing energy cost.

Based on the context above, the contributions of this paper are threefold:

1) A real-time framework for the PDISCO trading with heterogeneous DGOs.

2) A bilevel model for the PDISCO to achieve optimal trading strategies between real-time power exchanging and various types of the DGOs' bids.

3) A recast of the PDISCO's game-theoretic model to an MPEC through substituting the LL problems with an equivalent primal-dual approach.

The remainder of this paper is organized as follows. The real-time PDISCO-DGO trading framework is presented in Section II. Section III proposes the bilevel game-theoretic model of PDISCO trading with heterogeneous DGOs, and details the primal-dual solution approach. The results of the case studies are provided and discussed in Section IV. Finally, Section V concludes the paper with some relevant remarks.

\section{PDISCO-DGO REAL-TIME TRADING}

A real-time trading framework between PDISCO and heterogeneous DGOs is proposed in this section, as shown in Fig. 1. In the real-time trading process, at each time $t$, the profit-driven PDISCO has to make trading strategies on the procurements $\left(\lambda_{t(m, k, n)}^{B(1,2,3)}, P_{t(m, k, n)}^{B(1,2,3)}\right)$ from the DGOs and the transactions (selling or purchasing by $\lambda_{t}^{R T}, P_{t}^{R T}$ ) with the real-time market. When the PDISCO performs as an active producer, its offering price $\lambda_{t}^{R T}$ in the real-time market can be seen as the marginal price cleared from the transmission level at the interconnection point (main substation), while its offering volume $P_{t}^{R T}$ is realized as the surplus of power procurements after meeting the distribution network constraints.

Originated from the differing DGs' ownerships, the heterogeneous DGOs can be categorized into three types respectively indexed by $m, k$ and $n$, i.e. DGO $m$ with stochastic DGs, DGO $k$ with both stochastic DGs and SDs, and DGO $n$ with only SDs. The uncertainties of DGs can be represented by the scenario-based method [4]. At each time $t$ per scenario $\omega$ : Individual DGOs have the thorough knowledge of the cost $\left(C_{g}, C_{s}^{s d}, C_{s}^{s c}\right)$ and the production $\left(P_{t g \omega}^{D G}, P_{t s \omega}^{s d}, P_{t s \omega}^{s c}\right)$ pertaining to the related DGs. For profit maximization, each

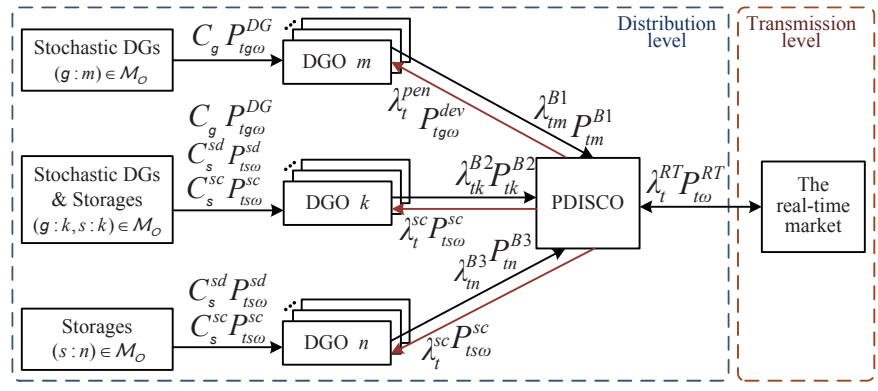

Fig. 1. Real-time trading framework of PDISCO with heterogeneous DGOs.

DGO should behave rationally to submit the appropriate bids to meet the PDISCO's request, implying the heterogeneous competition and peer pricing among the DGOs. For DGO $m$, if the actual power production can not meet the committed power generation, the power deviation $P_{t g \omega}^{d e v}$ occurs. In this case, DGO $m$ has to purchase $P_{t g \omega}^{d e v}$ from the PDISCO with a high penalty price $\lambda_{t}^{p e n}$. For DGO $k$, the self-owned SDs can assist in dealing with this kind of contingency. For both DGO $k$ and DGO $n$, the PDISCO supplies the charging power $P_{t s w}^{s c}$ for each affiliated SD with a contract price $\lambda_{t}^{s c}$.

\section{PROBLEM Formulation}

The PDISCO-DGO real-time trading problem can be formulated as a bilevel one-leader multi-follower-type gametheoretic model [3]. To embody the heterogeneous competition and peer pricing between three types of DGOs, an LL problem per DGO of a certain type indicates its rational bids to minimize its own minus-profit, see these categorized in (1)(3), respectively. While the UL problem (4) represents the PDISCO's minus-profit minimization.

\section{A. Assumptions}

The proposed bilevel model involves the following assumptions:

1) The PDISCO is assumed to only own and operate the network, and only one main substation is recognized as the exclusive interconnection point to the transmission network.

2) The real-time trading strategies of a single PDISCO are considered in this paper, including the power procurements from heterogeneous DGOs and power exchanging with the real-time market.

3) Only the active power is eligible to be traded between the PDISCO, DGOs and the real-time market, since the uniform reactive power market is not acknowledged.

4) Linking with $\lambda_{t(m, k, n)}^{B(1,2,3)}$, a DGO of a certain type can explicitly predict the impact of its bids (bidding prices and generation quantities), versus the PDISCO's offers (offering prices and power procurements).

5) We assume the Pay-as-bid (PAB) pricing [2] is the PDISCO-DGO trading mechanism, while the DGOs are further imposed to only be involved in the real-time trading with the PDISCO. One bid per DGO is allowed at each time $t$. 


\section{B. DGO LL Problems}

Note that the bidding prices and generation quantities, put forward by the differing DGOs from the LL problems, vary the PDISCO's procurement strategies in the UL problem. Therefore, the individual formulations for the three types of DGOs are enumerated below.

1) Type1: DGOs with stochastic DGs.

$$
\begin{aligned}
& \operatorname{Min}_{\Xi^{D G O 1}}-\sum_{t} \lambda_{t m}^{B 1} P_{t m}^{B 1}+\mathbb{E}\left[\sum_{t, g}\left(C_{g} P_{t g \omega}^{D G}+\lambda_{t}^{p e n} P_{t g \omega}^{d e v}\right)\right] \\
& \text { s.t. } \\
& P_{t m \omega}^{T P 1}=\sum_{g}\left(P_{t g \omega}^{D G}+P_{t g \omega}^{d e v}\right), \forall t, m, \omega \\
& Q_{t m \omega}^{T P 1}=\sum_{g}\left(Q_{t g \omega}^{D G}+Q_{t g \omega}^{d e v}\right), \forall t, m, \omega \\
& P_{t m \omega}^{T P 1} \leq P_{t m}^{B 1} \leq \sum_{g} \bar{P}_{t g}^{D G}, \forall t, m \\
& 0 \leq P_{t m \omega}^{T P 1} \leq \sum_{g} \bar{P}_{t g}^{D G}, \forall t, m, \omega \\
& 0 \leq Q_{t m \omega}^{T P 1} \leq \sum_{g} \bar{Q}_{t g}^{D G}, \forall t, m, \omega \\
& 0 \leq P_{t g \omega}^{d e v} \leq \bar{P}_{t g}^{D G}-P_{t g \omega}^{D G}, \forall t, g, \omega \\
& 0 \leq Q_{t g \omega}^{d e v} \leq \bar{Q}_{t g}^{D G}-Q_{t g \omega}^{D G}, \forall t, g, \omega
\end{aligned}
$$

where $\Xi^{D G O 1}=\left\{P_{t m}^{B 1}, P_{t m \omega}^{T P 1}, Q_{t m \omega}^{T P 1}, P_{t g \omega}^{d e v}, Q_{t g \omega}^{d e v}\right\}_{(g: m) \in \mathcal{M}_{O}}$ is the variable set of the LL problem for DGO $m$ pertaining to Type1.

The objective function (1a) is to minimize the minus-profit of DGO $m$, which consists of the minus-revenue of selling the committed generation quantities with the bidding prices, and the excepted cost of the stochastic DGs' productions plus power deviation penalties. At each time $t$ per scenario $\omega$ : Constraints (1b) and (1c) impose the total active/reactive power generated by DGO $m$, which are further limited through constraints (1e) and (1f). Constraints (1d) enforce the rational bidding quantity to cover each plausible realization of the DGs' generation. Constraints (1g) and (1h) express the limits of the power deviation caused by the generation uncertainty per DG.

2) Type2: DGOs with both stochastic DGs and SDs.

$$
\begin{aligned}
& \operatorname{Min}_{\Xi D G O 2}-\sum_{t} \lambda_{t k}^{B 2} P_{t k}^{B 2}+\mathbb{E}\left[\sum_{t, g} C_{g} P_{t g \omega}^{D G}\right. \\
& \left.+\sum_{t, s}\left(C_{s}^{s d} P_{t s \omega}^{s d}+\left(C_{s}^{s c}+\lambda_{t}^{s c}\right) P_{t s \omega}^{s c}\right)\right] \\
& \text { s.t. } \\
& P_{t k \omega}^{T P 2}=\sum_{g} P_{t g \omega}^{D G}+\sum_{s}\left(P_{t s \omega}^{s d}-P_{t s \omega}^{s c}\right), \\
& \forall t, k, \omega: \alpha_{t k \omega} \\
& Q_{t k \omega}^{T P 2}=\sum_{g} Q_{t g \omega}^{D G}+\sum_{s}\left(Q_{t s \omega}^{s d}-Q_{t s \omega}^{s c}\right), \\
& \forall t, k, \omega: \beta_{t k \omega} \\
& P_{t k}^{B 2} \geq P_{t k \omega}^{T P 2}, \forall t, k, \omega: \gamma_{t k \omega}
\end{aligned}
$$

$$
\begin{aligned}
& 0 \leq P_{t k}^{B 2} \leq \sum_{g} \bar{P}_{t g}^{D G}+\sum_{s} \bar{P}_{s}^{s d}, \forall t, k: \mu_{t k}^{-}, \mu_{t k}^{+} \\
& \sum_{g} \bar{P}_{t g}^{D G}-\sum_{s} \bar{P}_{s}^{s c} \leq P_{t k \omega}^{T P 2} \leq \sum_{g} \bar{P}_{t g}^{D G}+\sum_{s} \bar{P}_{s}^{s d}, \\
& \forall t, k, \omega: \psi_{t k \omega}^{-}, \psi_{t k \omega}^{+} \\
& \sum_{g} \bar{Q}_{t g}^{D G}-\sum_{s} \bar{Q}_{s}^{s c} \leq Q_{t k \omega}^{T P 2} \leq \sum_{g} \bar{Q}_{t g}^{D G}+\sum_{s} \bar{Q}_{s}^{s d}, \\
& \forall t, k, \omega: \rho_{t k \omega}^{-}, \rho_{t k \omega}^{+} \\
& E_{t+1, s, \omega}=E_{t, s, \omega}+\Delta t P_{t s \omega}^{s c} \eta_{s}^{s c}-\Delta t P_{t s \omega}^{s d} / \eta_{s}^{s d} \\
& \forall t, s, \omega: \zeta_{t s \omega} \\
& \underline{E}_{s} \leq E_{t s \omega} \leq \bar{E}_{s}, \forall t, s, \omega: \epsilon_{t s \omega}^{-}, \epsilon_{t s \omega}^{+} \\
& 0 \leq P_{t s \omega}^{s d} \leq \bar{P}_{s}^{s d}, \forall t, s, \omega: \theta_{t s \omega}^{-}, \theta_{t s \omega}^{+} \\
& 0 \leq P_{t s \omega}^{s c} \leq \bar{P}_{s}^{s c}, \forall t, s, \omega: \phi_{t s \omega}^{-}, \phi_{t s \omega}^{+} \\
& 0 \leq Q_{t s \omega}^{s d} \leq \bar{Q}_{s}^{s d}, \forall t, s, \omega: \sigma_{t s \omega}^{-}, \sigma_{t s \omega}^{+} \\
& 0 \leq Q_{t s \omega}^{s c} \leq \bar{Q}_{s}^{s c}, \forall t, s, \omega: \nu_{t s \omega}^{-}, \nu_{t s \omega}^{+}
\end{aligned}
$$

where $\Xi^{D G O 2}=\left\{P_{t k}^{B 2}, P_{t k \omega}^{T P 2}, Q_{t k \omega}^{T P 2}, P_{t s \omega}^{s d}, Q_{t s \omega}^{s d}, P_{t s \omega}^{s c}, Q_{t s \omega}^{s c}\right.$, $\left.E_{t s \omega}\right\}_{(g: k, s: k) \in \mathcal{M}_{O}}$ is the variable set of the LL problem for DGO $k$ regarding Type2.

The objective (2a) indicates the minus-profit minimization of DGO $k$, i.e., the minus-revenue of selling the committed supplies to the PDISCO plus the excepted cost of the DGs' and SDs' productions. At each time $t$ per scenario $\omega$ : Constraints (2b), (2c), (2f) and (2g) identify the active/reactive power availability of DGO $k$. To cover each plausible realization of the DGs' generation, constraints (2d) impose the bidding quantity $P_{t k}^{B 2}$ towards a rational bid. Considering only positive bids have the possibility to be accepted by the PDISCO, the bidding quantity bounds are further restricted by (2e). The residual energy of each SD $s$ is enforced by constraints (2h) and bounded by constraints (2i), while the capabilities of the discharged/charged active/reactive power are constrained via $(2 \mathrm{j}),(2 \mathrm{k}),(2 \mathrm{l})$ and $(2 \mathrm{~m})$. Furthermore, the dual variables for each group of constraints are indicated correspondingly, separated by a colon.

3) Type3: DGOs with only SDs.

$$
\begin{aligned}
& \operatorname{Min}_{\Xi D G O 3}-\sum_{t} \lambda_{t n}^{B 3} P_{t n}^{B 3} \\
& +\mathbb{E}\left[\sum_{t, s}\left(C_{s}^{s d} P_{t s \omega}^{s d}+\left(C_{s}^{s c}+\lambda_{t}^{s c}\right) P_{t s \omega}^{s c}\right)\right] \\
& \text { s.t. } \\
& P_{t n \omega}^{T P 3}=\sum_{s}\left(P_{t s \omega}^{s d}-P_{t s \omega}^{s c}\right), \forall t, n, \omega \\
& Q_{t n \omega}^{T P 3}=\sum_{s}\left(Q_{t s \omega}^{s d}-Q_{t s \omega}^{s c}\right), \forall t, n, \omega \\
& P_{t n}^{B 3} \geq P_{t n \omega}^{T P 3}, \forall t, n, \omega \\
& 0 \leq P_{t n}^{B 3} \leq \sum_{s} \bar{P}_{s}^{s d}, \forall t, n \\
& -\sum_{s} \bar{P}_{s}^{s c} \leq P_{t n \omega}^{T P 3} \leq \sum_{s} \bar{P}_{s}^{s d}, \forall t, n, \omega \\
& -\sum_{s} \bar{Q}_{s}^{s c} \leq Q_{t n \omega}^{T P 2} \leq \sum_{s} \bar{Q}_{s}^{s d}, \forall t, n, \omega \\
& E_{t+1, s, \omega}=E_{t, s, \omega}+\Delta t P_{t s \omega}^{s c} \eta_{s}^{s c}-\Delta t P_{t s \omega}^{s d} / \eta_{s}^{s d},
\end{aligned}
$$




$$
\begin{aligned}
& \forall t, s, \omega \\
& \underline{E}_{s} \leq E_{t s \omega} \leq \bar{E}_{s}, \forall t, s, \omega \\
& 0 \leq P_{t s \omega}^{s d} \leq \bar{P}_{s}^{s d}, \forall t, s, \omega \\
& 0 \leq P_{t s \omega}^{s c} \leq \bar{P}_{s}^{s c}, \forall t, s, \omega \\
& 0 \leq Q_{t s \omega}^{s d} \leq \bar{Q}_{s}^{s d}, \forall t, s, \omega \\
& 0 \leq Q_{t s \omega}^{s c} \leq \bar{Q}_{s}^{s c}, \forall t, s, \omega
\end{aligned}
$$

where $\Xi^{D G O 3}=\left\{P_{t n}^{B 3}, P_{t n \omega}^{T P 3}, Q_{t n \omega}^{T P 3}, P_{t s \omega}^{s d}, Q_{t s \omega}^{s d}, P_{t s \omega}^{s c}, Q_{t s \omega}^{s c}\right.$, $\left.E_{t s \omega}\right\}_{(s: n) \in \mathcal{M}_{O}}$ is the variable set of the LL problem for DGO $n$ corresponding to Type3.

Excluding the DGs' stochastic productions given in (2), we can obtain the constraints (3b)-(3m) for DGO $n$ with only SDs and the objective (3a) to minimize its minus-profit.

\section{PDISCO UL Problem}

Note that the UL problem and the LL problems are interrelated with each other. The decisions (offering prices and power procurements) made by the UL problem directly impact the DGOs' profit in the LL problems. Thus, the formulation of the UL problem constitutes the PDISCO's offer strategies and physical network constraints, as well as the bid arguments from the heterogeneous DGOs.

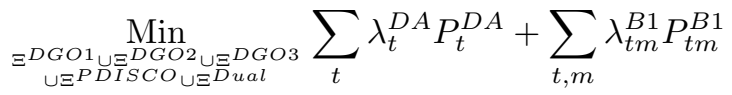

$$
\begin{aligned}
& +\sum_{t, k} \lambda_{t k}^{B 2} P_{t k}^{B 2}+\sum_{t, n} \lambda_{t n}^{B 3} P_{t n}^{B 3}+\mathbb{E}\left[\sum_{t} \lambda_{t}^{R T} P_{t \omega}^{R T}\right. \\
& +\sum_{t, d} \lambda_{t}^{\text {shed }} P_{t d \omega}^{\text {shed }}-\sum_{t, g} \lambda_{t}^{p e n} P_{t g \omega}^{d e v}-\sum_{t, s} \lambda_{t}^{s c} P_{t s \omega}^{s c} \\
& \left.-\sum_{t, d} \lambda_{t}^{D}\left(P_{t d}^{D}-P_{t d \omega}^{S h e d}\right)\right] \\
& \text { s.t. } \\
& \Upsilon_{t m}^{T P 1} \sum_{(g: m) \in \mathcal{M}_{O}} C_{g} \leq \lambda_{t m}^{B 1} \leq \lambda_{t}^{R T}, \forall t, m \\
& \Upsilon_{t k}^{T P 2}\left(\sum_{(g: k) \in \mathcal{M}_{O}} C_{g}+\sum_{(s: k) \in \mathcal{M}_{O}}\left(C_{s}^{s d}+C_{s}^{s c}+\lambda_{t}^{s c}\right)\right) \\
& \leq \lambda_{t k}^{B 2} \leq \lambda_{t}^{R T}, \forall t, k \\
& \Upsilon_{t n}^{T P 3} \sum_{(s: n) \in \mathcal{M}_{O}}\left(C_{s}^{s d}+C_{s}^{s c}+\lambda_{t}^{s c}\right) \\
& \leq \lambda_{t n}^{B 3} \leq \lambda_{t}^{R T}, \forall t, n
\end{aligned}
$$

For the main substation (reference bus 1):

$$
\begin{aligned}
& \sum_{(g: 1) \in \mathcal{M}_{G}}\left(P_{t 1 \omega}^{D G}-P_{t 1 \omega}^{d e v}\right)+\sum_{(s: 1) \in \mathcal{M}_{S}}\left(P_{t 1 \omega}^{s d}-P_{t 1 \omega}^{s c}\right) \\
+ & P_{t}^{D A}+P_{t \omega}^{R T}+P_{t 1 \omega}^{s h e d}-P_{t 1}^{D}=\sum_{1 j \in \Lambda} P_{t, 1 j, \omega}^{f}, \forall t, \omega(4 \mathrm{e}) \\
& \sum_{(g: 1) \in \mathcal{M}_{G}}\left(Q_{t 1 \omega}^{D G}-Q_{t 1 \omega}^{d e v}\right)+\sum_{(s: 1) \in \mathcal{M}_{S}}\left(Q_{t 1 \omega}^{s d}-Q_{t 1 \omega}^{s c}\right) \\
& +Q_{t}^{D A}+Q_{t \omega}^{R T}+Q_{t 1 \omega}^{s h e d}-Q_{t 1}^{D}+Q_{t 1 \omega}^{C} \\
= & \sum_{1 j \in \Lambda} Q_{t, 1 j, \omega}^{f}, \forall t, \omega
\end{aligned}
$$

$$
\begin{aligned}
& e_{t 1 \omega}=1, \forall t, \omega \\
& \delta_{t 1 \omega}=0, \forall t, \omega \\
& \left(P_{t}^{D A}+P_{t \omega}^{R T}\right)^{2}+\left(Q_{t}^{D A}+Q_{t \omega}^{R T}\right)^{2} \leq \bar{S}^{2}, \forall t, \omega
\end{aligned}
$$

For the other buses:

$$
\begin{aligned}
& \sum_{(g: i) \in \mathcal{M}_{G}}\left(P_{t g \omega}^{D G}-P_{t g \omega}^{d e v}\right)+\sum_{(s: i) \in \mathcal{M}_{S}}\left(P_{t s \omega}^{s d}-P_{t s \omega}^{s c}\right) \\
+ & P_{t d \omega}^{s h e d}-P_{t d}^{D}=\sum_{i j \in \Lambda} P_{t, i j, \omega}^{f}, \forall t, i_{(d: i) \in \mathcal{M}_{D}}, \omega \\
& \sum_{(g: i) \in \mathcal{M}_{G}}\left(Q_{t g \omega}^{D G}-Q_{t g \omega}^{d e v}\right)+\sum_{(s: i) \in \mathcal{M}_{S}}\left(Q_{t d \omega}^{s d}-Q_{t d \omega}^{s c}\right) \\
+ & Q_{t d \omega}^{s h e d}-Q_{t d}^{D}+Q_{t i \omega}^{C}=\sum_{i j \in \Lambda} Q_{t, i j, \omega}^{f}, \forall t, i_{(d: i) \in \mathcal{M}_{D}}, \omega
\end{aligned}
$$

$$
\begin{aligned}
& P_{t, i j, \omega}^{f}=-\tau_{i} e_{t i \omega}^{2} G_{i j}+e_{t i \omega} e_{t j \omega}\left[G_{i j} \cos \left(\delta_{t i \omega}-\delta_{t j \omega}\right)\right. \\
& \left.+B_{i j} \sin \left(\delta_{t i \omega}-\delta_{t j \omega}\right)\right], \forall t, i j \in \Lambda, \omega \\
& Q_{t, i j, \omega}^{f}=\tau_{i} e_{t i \omega}^{2} B_{i j}+e_{t i \omega} e_{t j \omega}\left[G_{i j} \sin \left(\delta_{t i \omega}-\delta_{t j \omega}\right)\right. \\
& \left.-B_{i j} \cos \left(\delta_{t i \omega}-\delta_{t j \omega}\right)\right]-0.5 b_{i j}, \forall t, i j \in \Lambda, \omega \\
& -\pi \leq \delta_{t i \omega} \leq \pi, \forall t, i, \omega \\
& \underline{e}_{i} \leq e_{t i \omega} \leq \overline{e_{i}}, \forall t, i, \omega \\
& \left(P_{t, i j, \omega}^{f}\right)^{2}+\left(Q_{t, i j, \omega}^{f}\right)^{2} \leq\left(\bar{S}_{i j}\right)^{2}, \forall t, i j \in \Lambda, \omega \\
& 0 \leq P_{t d \omega}^{s h e d} \leq P_{t d}^{D}, \forall t, d, \omega \\
& 0 \leq Q_{t d \omega}^{s h e d} \leq Q_{t d}^{D}, \forall t, d, \omega \\
& Q_{i}^{C} \leq Q_{t i \omega}^{C} \leq \bar{Q}_{i}^{C}, \forall t, i, \omega \\
& P_{t d}^{D} Q_{t d \omega}^{s h e d}-P_{t d \omega}^{s h e d} Q_{t d}^{D}=0, \forall t, d, \omega \\
& P_{t d}^{D} Q_{t d \omega}^{d e v}-P_{t d \omega}^{d e v} Q_{t d}^{D}=0, \forall t, d, \omega \\
& P_{t m}^{B 1}, P_{t g \omega}^{d e v}, Q_{t g \omega}^{d e v} \in \arg (1)_{(g: m) \in \mathcal{M}_{O}} \\
& P_{t k}^{B 2}, P_{t s \omega}^{s d}, Q_{t s \omega}^{s d}, P_{t s \omega}^{s c}, Q_{t s \omega}^{s c} \in \arg (2)_{(g: k, s: k) \in \mathcal{M}_{O}} \\
& P_{t n}^{B 3}, P_{t s \omega}^{s d}, Q_{t s \omega}^{s d}, P_{t s \omega}^{s c}, Q_{t s \omega}^{s c} \in \arg (3)_{(s: n) \in \mathcal{M}_{O}}
\end{aligned}
$$

where $\Xi^{P D I S C O}=\left\{\lambda_{t m}^{B 1}, \lambda_{t k}^{B 2}, \lambda_{t n}^{B 3}, P_{t \omega}^{R T}, Q_{t \omega}^{R T}, Q_{t i \omega}^{C}, P_{t d \omega}^{s h e d}\right.$, $\left.Q_{t d \omega}^{s h e d}, P_{t, i j, \omega}^{f}, Q_{t, i j, \omega}^{f}, \delta_{t i \omega}, e_{t i \omega}\right\}$ is the variable set of the UL PDISCO problem. $\Xi^{D U A L}$ is the set of dual variables.

The objective (4a) of the UL problem is to minimize the PDISCO's minus-profit, which consists of two aspects. The first aspect contains the purchases from the day-ahead market and the procurements from the various types of DGOs. The second aspect is the expected minus-profit according to the power exchanging from the real-time market, the penalty of load-shedding, the DGOs' payment of DGs' power deviation and SDs' power charging, and the minus-revenue of electricity sales to the demands. At each time $t$ : The bidding prices submitted by various DGOs include their own profit guarantee scheme, and these prices are considered as the lower bounds from the PDISCO perspective in constraints (4b)-(4d), while the upper bounds are imposed as the real-time market price. AC power flow is yielded to formulate the PDISCO's realtime operation model. Constraints (4e) and (4f) represent the $\mathrm{AC}$ power balance at the reference bus (main substation), which maintains the voltage value and voltage angle at a constant level through constraints (4g) and (4h). The capacity 
limit of the main substation is identified by constraints (4i). Constraints (4j) and (4k) enforce the $\mathrm{AC}$ power balance at the other buses, in which the voltage angle and voltage value are bounded by constraints $(4 \mathrm{n})$ and $(4 \mathrm{o})$. Constraints (4l) and (4m) identify the AC power flow through feeder $i$ $j$. Constraints (4p) specify the capacity limits of individual feeders. Constraints $(4 q)$ and $(4 r)$ indicate the load-shedding limits. Constraints (4s) depict the capacity bounds of each compensator. Constraints (4t) maintain a constant demand power factor, if load-shedding occurs. Constraints $(4 \mathrm{u})$ keep the generation power factor stable when the power deviation of a stochastic DG arises. Observe that the PDISCO offering prices, i.e., $\lambda_{t(m, k, n)}^{B(1,2,3)}$, are UL decision variables treated as parameters in the LL problems. Concerning the formulations categorized in Section III-B, constraints (4v), (4w) and (4x) indicate the heterogeneous DGOs within Types1-3 intend to maximize their own profits, individually.

\section{D. $M P E C$}

Note that the UL PDISCO problem is nonlinear and nonconvex, while the LL DGOs' problems are linear and thus convex. To transform the proposed bilevel model into a singlelevel optimization problem, the varied DGOs' problems can be replaced by their first-order optimality conditions, which renders an MPEC. Here, two alternative approaches are available for the reformulation of this problem, i.e., Karush-KuhnTucker (KKT) conditions and primal-dual approach.

In general, the primal-dual approach renders a mathematical program with primal and dual constraints (MPPDC), which is more tractable and efficient for off-the-shelf branch-andcut software than its associated KKT conditions [5] [23]. Thus, the primal-dual approach is employed in this paper. For brevity, the DGO $k$ within Type 2 of the LL problems is taken as an illustrative example to carry out the MPPDC transformation, as shown in (5). Constraints (5a)-(5h) are the dual constraints of the primal constraints $(2 \mathrm{~b})-(2 \mathrm{~m})$. Constraint (5i) is the associated strong duality equality, which ensures the equality of the primal and dual objective values, one per DGO $k$. Subsequently, the similar MPPDC transformations can be applied to the DGOs characterized in Type1 and Type3, respectively.

$$
\begin{aligned}
& -\lambda_{t k}^{B 2}-\gamma_{t k \omega}+\mu_{t k}^{+}-\mu_{t k}^{-}=0, \forall t, k \\
& \alpha_{t k \omega}+\gamma_{t k \omega}+\psi_{t k \omega}^{+}-\psi_{t k \omega}^{-}=0, \forall t, k, \omega \\
& \beta_{t k \omega}+\rho_{t k \omega}^{+}-\rho_{t k \omega}^{-}=0, \forall t, k, \omega \\
& C_{s}^{s d}-\alpha_{t k \omega}+\Delta t \zeta_{t s \omega} / \eta_{s}^{s d}+\theta_{t s \omega}^{+}-\theta_{t s \omega}^{-}=0, \\
& \forall t, s_{(s: k) \in \mathcal{M}_{O}}, \omega \\
& -\beta_{t k \omega}+\sigma_{t s \omega}^{+}-\sigma_{t s \omega}^{-}=0, \forall t, s(s: k) \in \mathcal{M}_{O}, \omega \\
& C_{s}^{s c}+\lambda_{t}^{s c}+\alpha_{t k \omega}-\Delta t \zeta_{t s \omega} \eta_{s}^{s c}+\phi_{t s \omega}^{+}-\phi_{t s \omega}^{-}=0, \\
& \forall t, s_{(s: k) \in \mathcal{M}_{O}}, \omega \\
& \beta_{t k \omega}+\nu_{t s \omega}^{+}-\nu_{t s \omega}^{-}=0, \forall t, s_{(s: k) \in \mathcal{M}_{O}}, \omega \\
& \zeta_{t s \omega}-\zeta_{t-1, s, \omega}+\epsilon_{t s \omega}^{+}-\epsilon_{t s \omega}^{-}=0, \forall t, s_{(s: k) \in \mathcal{M}_{O}}, \omega \\
& \sum_{t} \lambda_{t k}^{B 2} P_{t k}^{B 2}+\sum_{t} \mu_{t k}\left(\sum_{g} \bar{P}_{t g}^{D G}+\sum_{s} \bar{P}_{s}^{s d}\right)+
\end{aligned}
$$

$$
\begin{gathered}
\text { is } \\
\text { and } \\
\text { Dets } \\
\text { the }
\end{gathered}
$$

$$
\mathbb{E}\left[-\sum_{t, g} C_{g} P_{t g \omega}^{D G}-\sum_{t, s}\left(C_{s}^{s d} P_{t s \omega}^{s d}+\left(C_{s}^{s c}+\lambda_{t}^{s c}\right) P_{t s \omega}^{s c}\right)\right.
$$


day. The related mappings and parameters of the assorted DGOs with DGs are described in Table I. For simplicity, the SDs' discharging/charging limits and costs are identical with a unified efficiency 0.9 , while the contracted charging price $\lambda_{t}^{s c}$ can be assumed as half the price of the day-ahead prices. The profit guarantee factors $\Upsilon_{t(m, n, k)}^{T P(1,2,3)}$ follow the rate of change of the real-time prices with the individual base-values 14, 6 and 7. The power factors are recognized as $0.90 / \mathrm{WT}, 0.95 / \mathrm{PV}$, and $0.99 / \mathrm{SD}$. For the PDISCO, the real-time demand $P_{t d}^{D}$ and day-head purchases $P_{t}^{D A}$ are shown in Table II, in which the prices $\lambda_{t}^{D A}, \lambda_{t}^{R T}$ and $\lambda_{t}^{D}$ are estimated by the NordPool [28] prices. The penalty price $\lambda_{t}^{\text {pen }}$ is also claimed for the potential power deviation caused by the DGOs' output mismatching the committed capacity. In addition, the load-shedding price $\lambda_{t}^{\text {shed }}$ is considered as 200 times as $\lambda_{t}^{R T}$. The other parameters can be found in [25].

TABLE I

INPUT PARAMETERS OF DGOS AND DGS

\begin{tabular}{|c|c|c|c|c|c|}
\hline Type & $\begin{array}{c}\text { DGO } \\
(m, k, n)\end{array}$ & $\begin{array}{c}\mathcal{M}_{G}, \mathcal{M}_{S} \\
(g: i, s: i)\end{array}$ & $\begin{array}{c}\bar{P}_{t g}^{D G}, \\
\bar{P}_{s}^{s d}, \bar{P}_{s c}^{s c} \\
{[\mathrm{~kW}]}\end{array}$ & $\frac{\underline{E}_{s}, \bar{E}_{s}}{[\mathrm{kWh}]}$ & $\begin{array}{c}C_{g}, \\
C_{s}^{s d}, C_{s}^{s c} \\
{\left[10^{-2} € / \mathrm{kW}\right]}\end{array}$ \\
\hline \multirow{4}{*}{ Type 1} & \multirow{2}{*}{$m=1$} & WT1:21 & 300 & - & 1.33 \\
\hline & & WT2:7 & 300 & - & 1.47 \\
\hline & \multirow[b]{2}{*}{$m=2$} & WT3:12 & 300 & - & 1.60 \\
\hline & & PV1:7 & 300 & - & 1.87 \\
\hline \multirow{4}{*}{ Type2 } & \multirow[b]{2}{*}{$k=1$} & WT4:25 & 300 & - & 1.73 \\
\hline & & SD1:30 & 300 & 50,400 & 2.27 \\
\hline & \multirow[b]{2}{*}{$k=2$} & PV2:19 & 300 & - & 2.00 \\
\hline & & SD2:8 & 300 & 50,400 & 2.33 \\
\hline \multirow{4}{*}{ Type 3} & \multirow[b]{2}{*}{$n=1$} & SD3:24 & 600 & 100,800 & 2.00 \\
\hline & & SD4:29 & 300 & 50,400 & 2.07 \\
\hline & \multirow[b]{2}{*}{$n=2$} & SD5:32 & 300 & 50,400 & 2.13 \\
\hline & & SD6:14 & 300 & 50,400 & 2.20 \\
\hline
\end{tabular}

TABLE II

Essential InPut Parameters For PDISCO-DGO Trading Model

\begin{tabular}{c|c|c|c|c|c|c}
\hline $\begin{array}{c}t \\
{[\text { Hour }]}\end{array}$ & $\begin{array}{c}\lambda_{t}^{D A} \\
{[€ / \mathrm{kW}]}\end{array}$ & $\begin{array}{c}P_{t}^{D A} \\
{[\mathrm{~kW}]}\end{array}$ & $\begin{array}{c}\lambda_{t}^{R T} \\
{[€ / \mathrm{kW}]}\end{array}$ & $\begin{array}{c}\lambda_{t}^{D} \\
{[€ / \mathrm{kW}]}\end{array}$ & $\begin{array}{c}P_{t d}^{D} \\
{[\mathrm{~kW}]}\end{array}$ & $\begin{array}{c}\lambda_{t}^{p e n} \\
{[€ / \mathrm{kW}]}\end{array}$ \\
\hline 1 & 0.10 & 1057.63 & 0.16 & 0.20 & 1114.50 & 0.28 \\
\hline 2 & 0.11 & 1270.13 & 0.17 & 0.21 & 1188.80 & 0.30 \\
\hline 3 & 0.12 & 1401.27 & 0.19 & 0.23 & 1300.25 & 0.33 \\
\hline 4 & 0.10 & 1057.63 & 0.16 & 0.20 & 1114.50 & 0.28 \\
\hline 5 & 0.13 & 1258.24 & 0.21 & 0.27 & 1486.00 & 0.37 \\
\hline 6 & 0.14 & 1320.65 & 0.22 & 0.28 & 1560.30 & 0.39 \\
\hline 7 & 0.16 & 2114.18 & 0.26 & 0.32 & 1783.20 & 0.45 \\
\hline 8 & 0.17 & 2283.58 & 0.27 & 0.34 & 1894.65 & 0.48 \\
\hline 9 & 0.22 & 2907.70 & 0.35 & 0.43 & 2414.75 & 0.61 \\
\hline 10 & 0.24 & 2586.35 & 0.39 & 0.49 & 2711.95 & 0.68 \\
\hline 11 & 0.33 & 3213.82 & 0.52 & 0.65 & 3640.70 & 0.91 \\
\hline 12 & 0.37 & 3646.99 & 0.59 & 0.73 & 4086.50 & 1.03 \\
\hline 13 & 0.37 & 3606.12 & 0.59 & 0.73 & 4086.50 & 1.03 \\
\hline 14 & 0.30 & 3888.46 & 0.48 & 0.60 & 3343.50 & 0.84 \\
\hline 15 & 0.27 & 3487.24 & 0.43 & 0.53 & 2972.00 & 0.75 \\
\hline 16 & 0.24 & 3086.02 & 0.38 & 0.48 & 2674.80 & 0.67 \\
\hline 17 & 0.28 & 3799.30 & 0.45 & 0.57 & 3157.75 & 0.79 \\
\hline 18 & 0.30 & 3788.16 & 0.48 & 0.60 & 3343.50 & 0.84 \\
\hline 19 & 0.35 & 4495.86 & 0.56 & 0.70 & 3900.75 & 0.98 \\
\hline 20 & 0.37 & 3687.85 & 0.59 & 0.73 & 4086.50 & 1.03 \\
\hline 21 & 0.37 & 3442.66 & 0.59 & 0.73 & 4086.50 & 1.03 \\
\hline 22 & 0.27 & 3457.52 & 0.43 & 0.53 & 2972.00 & 0.75 \\
\hline 23 & 0.17 & 2053.25 & 0.27 & 0.33 & 1857.50 & 0.47 \\
\hline 24 & 0.13 & 1614.88 & 0.21 & 0.27 & 1486.00 & 0.37 \\
\hline
\end{tabular}

The results of power exchanging in the real-time market are shown in Fig. 2. As expected, the PDISCO behaves as an active producer to provide productions at certain periods, e.g., scenario-based $(\omega=1)$ power exchanging in hours $8-9,14-15$, and 17-19. Hours $(8-9,14-15)$ reflect that the PDISCO has the ability to sell the excess power reversely to the transmission level in medium-price areas. Observe that two peaks appear at hours 11-13 and 19-21, while the results of the power transactions are distinct. During hours 11-13, the PDISCO acquires only a little power, implying the PDISCO executes the proper strategy to avoid the volatile real-time prices and insufficient day-ahead purchases with the DGOs' bids. Hour 19 overlaps with the system's peak and the PDISCO's active performance, indicating the PDISCO's arbitrage capability to facilitate the sales strategy in a profitable high-price time slot with abundant generation from DGOs and sufficient day-ahead dealings. Running through the low-price intervals, e.g., hours 1-7 and 23-24, the PDISCO strategically launches large-volume procurements to increase the revenue by selling charging power to the SD-equipped DGOs.

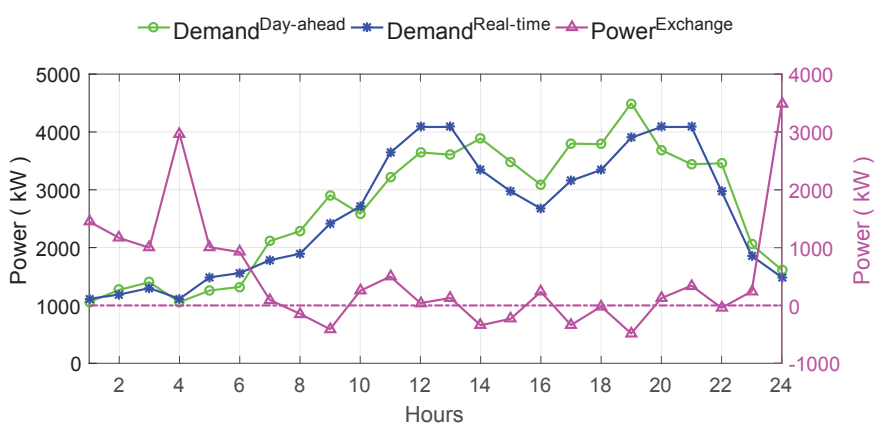

Fig. 2. Power exchanging in the real-time market.

Interacting with the PDISCO, as profit-driven entities, the individual DGOs perform rationally to submit bids $\left(\lambda_{t(m, k, n)}^{B(1,2,3)}\right.$, $\left.P_{t(m, k, n)}^{B(1,2,3)}\right)$, resulting in the PDISCO's offering prices and power procurements, as shown in Fig. 3 in detail. As observed, in Fig. 3 (a), the DGOs with only stochastic DGs continuously obtain offers with lower prices, versus large amounts. Since the DGs' power deviation against committed generation is inevitable, the DGOs' repurchases negatively accompany the offers per hour, e.g., scenario-based $(\omega=1)$ power deviations. The minor difference between the WT-PV and the WT-WT DGOs is that the later bids are at lower prices with higher quantities. In contrast, the WT-SD and PV-SD DGOs generally bid at higher prices, as shown in Fig. 3 (b). However, the generation quantities are not comparable, reduced critically for WT-SD DGO, and even declined in some periods for PV-SD DGO, although the SDs are functional to cover the hourly power deviation. Fig. 3 (c) reveals that the two SD-SD DGOs take similar actions to respond the DISCO's request with the highest bidding prices only at the peaks. Note that the generation quantities are quite limited, the DGO with higher capacity leads to a higher competitiveness.

In other words, the characteristics of heterogeneous competition and peer pricing among DGOs have been achieved in the proposed methodology. Accordingly, the profits of each participant per hour are obtained and shown in Fig. 4, and the daily profits are summarized in Profit1 of Table III.

Considering each Type1 DGO's profit mainly depends on the owned DGs' total capacity, while the stochastic outputs are 


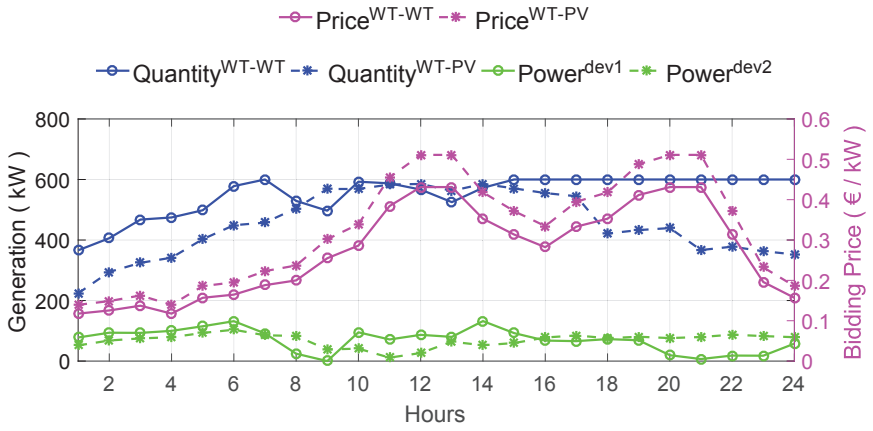

(a) DGOs of Type 1

- - Price $^{\text {WT-SD }- \text { Price }}{ }^{\mathrm{PV}-\mathrm{SD}}$

$\rightarrow$ QuantityWT-SD -* QuantityPV-SD

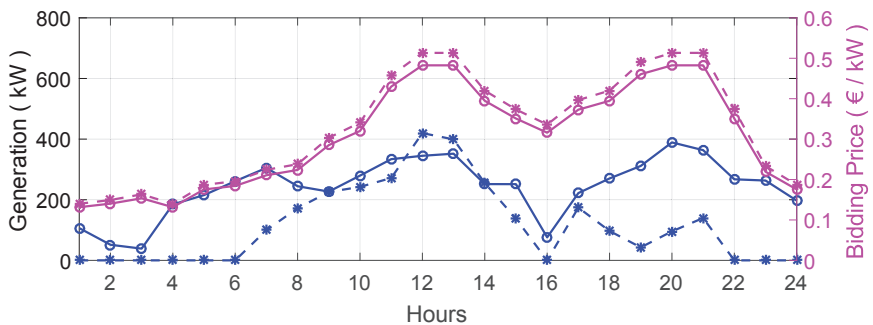

(b) DGOs of Type2

$\rightarrow$ Price $^{\text {SD-SD1 }}$-*-2 Price ${ }^{\text {SD-SD2 }}$

$\rightarrow$ Quantity SD-SD1 -* Quantity SD-SD2

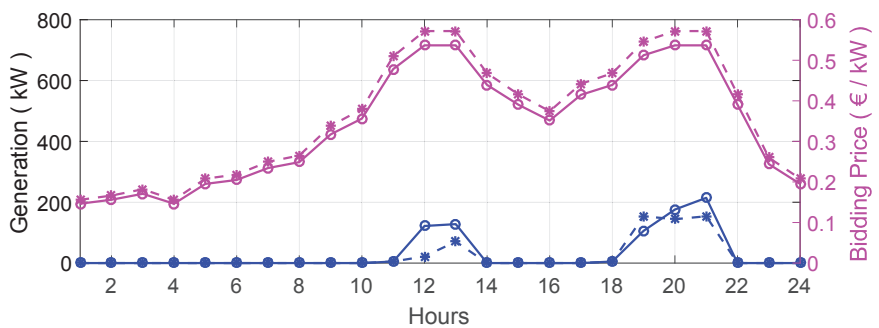

(c) DGOs of Type3

Fig. 3. The PDISCO's hourly offers for the heterogeneous DGOs.

uncontrollable and formulated as scenario-based parameters, we further focus on the SD's impacts for the PDISCO-DGO trading decisions by resetting the SDs' bounds of discharging/charging power and residual energy in the other cases. Thus, the discussions above are seen as Case 1 .

Keeping the other parameters' settings above, the additional cases are regarded as follows: Case 2 reduces each SD's $\bar{P}_{s}^{s d}$ and $\bar{P}_{s}^{s c}$ six times as Case 1, while these are increased two times in Case 3. Furthermore, on the basis of Case 1, the respective SDs' $\underline{E}_{s}, \bar{E}_{s}$ are with five times increment in Case 4 and two times decrement in Case 5. The relevant profits are listed as Profit2-5 in Table III.

Observe that the SDs with higher residual energy can bring more profit to the owners, compared with Profi4 and Profit5, but provide quite limited effort for the PDISCO and other DGOs. The variance between Profit 2 and Profit 3 indicates that higher capacity of DGs renders a dramatic profit increment for the PDISCO. The DGOs within Type1,2 are more competitive than the SDs' owners, who perform in a steady mode. However, it is quite profitable for the Type2 DGOs by eliminating trading uncertainties with SDs. Thus, to improve

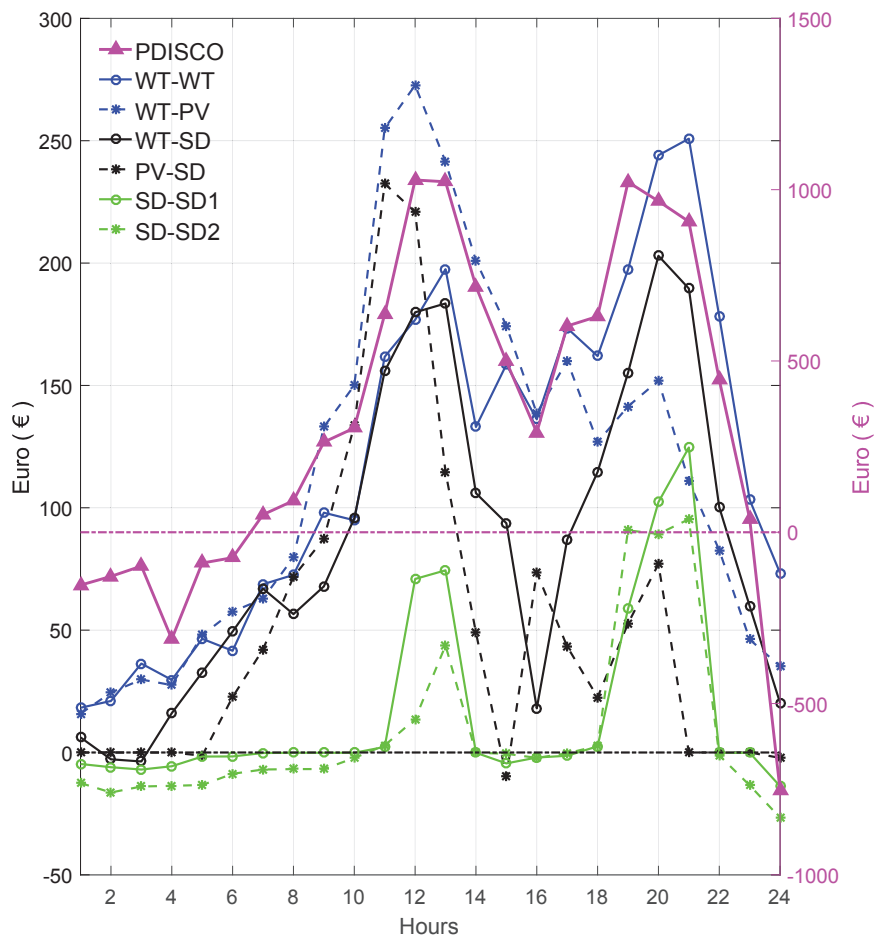

Fig. 4. Hourly profit of the PDISCO and individual DGOs.

the profitability for any type of DGO, SDs with higher residue and high capacity are the best option.

TABLE III

DAILY PROFIT OF THE PDISCO AND INDIVIDUAL DGOS

\begin{tabular}{c|c|c|c|c|c|c}
\hline $\begin{array}{c}\text { Parti- } \\
\text { cipant }\end{array}$ & $\begin{array}{c}\text { Profit1 } \\
{[€]}\end{array}$ & $\begin{array}{c}\text { Profit2 } \\
{[€]}\end{array}$ & $\begin{array}{c}\text { Profit3 } \\
{[€]}\end{array}$ & $\begin{array}{c}\text { Profit4 } \\
{[€]}\end{array}$ & $\begin{array}{c}\text { Profit5 } \\
{[€]}\end{array}$ & $\begin{array}{c}\text { Profit6 } \\
{[€]}\end{array}$ \\
\hline PDISCO & 7125.45 & 3253.33 & 11750.62 & 7484.41 & 6136.00 & 37566.29 \\
\hline WT-WT & 2589.67 & 475.77 & 5716.36 & 2588.96 & 2547.32 & 2576.13 \\
\hline WT-PV & 2493.79 & 460.87 & 5432.55 & 2438.55 & 2461.55 & 2421.52 \\
\hline WT-SD & 1848.77 & 399.80 & 3272.46 & 2030.27 & 1773.29 & 1822.10 \\
\hline PV-SD & 1108.76 & 283.51 & 2597.36 & 1315.85 & 1054.24 & 1110.28 \\
\hline SD-SD1 & 348.49 & 341.24 & 351.49 & 1040.67 & 186.95 & 410.36 \\
\hline SD-SD2 & 203.76 & 198.19 & 209.16 & 612.87 & 109.04 & 276.88 \\
\hline
\end{tabular}

\section{B. 119-bus Distribution Network}

The 119-bus network [26], [27] is modified to further test the scalability of the proposed approach. The parameters are set as Case 6. Particularly, individual demands are proportionately adjusted to follow the variation of the corresponding data in Section IV-A Case 1. The mappings of DGs and buses are WT1:7, WT2:19, WT3:66, WT4:110, PV1:33, PV2:89, SD1:40, SD2:78, SD3:10, SD4:116, SD5:29, and SD6:103. The other system-wide parameters remain the same as in Section IV-A.

The profit results are enumerated as Profit6 in Table III. Each DGO's profit is nearly consistent as in Case 1, whereas the minor differences can be caused by the differing layout of the physical network. The PDISCO's daily profit is increased by $427 \%$, primarily from sales revenue, since the demand is considerably high. 


\section{Computational Issue}

All cases are carried out on a $3.6 \mathrm{GHz}$ Intel Core i7 processor with $16 \mathrm{~GB}$ of RAM and 64-bit Windows 7 system, and solved by CONOPT3 with GAMS 24.4.1 [24].

Table IV summarizes the computational time for solving the problem corresponding to each case. Note that the computational burden increases significantly with the scale and complexity of the distribution network. However, it is also worth noting that the computational performance is acceptable for a hourly-based trading.

TABLE IV

Computational Time of EACH CAsE

\begin{tabular}{c|c|c|c}
\hline Case & CPU time $(\mathrm{s})$ & Case & CPU time (s) \\
\hline Case 1 & 498.63 & Case 4 & 499.70 \\
\hline Case 2 & 489.35 & Case 5 & 500.38 \\
\hline Case 3 & 503.22 & Case 6 & 2761.03 \\
\hline
\end{tabular}

\section{CONCLUSiON}

This paper proposes a bilevel game-theoretic model to investigate the PDISCO's real-time trading strategies between the type-oriented DGOs and the transmission-level market. Three types of DGOs and a real-time trading framework are well defined to enhance competitiveness, as in the distributionlevel market environment. Accompanying the UL PDISCO's optimal decisions, an LL DGO's problem also achieves its goal for profit maximization with the rational multi-period bids. With the primal-dual approach, the proposed model is reformulated to a solvable MPEC. The numerical results of the case studies successfully illustrate the heterogeneous competition and peer pricing characteristics of the DGOs, also demonstrate the PDISCO's trading strategies are suitable and effective.

\section{REFERENCES}

[1] NewYorkState, "Reforming the energy vision," NYS Department of Public Service, 2014.

[2] Q. Wang, C. Zhang, Y. Ding, G. Xydis, J. Wang, and J. Østergaard, "Review of real-time electricity markets for integrating distributed energy resources and demand response," Appl. Energy, vol. 138, pp. 695-706, 2015.

[3] D. Fudenberg and J. Tirole, Game theory. Cambridge, Massachusetts: The MIT Press, 1991.

[4] Z. Wang, B. Chen, J. Wang, M. M. Begovic, and C. Chen, "Coordinated energy management of networked microgrids in distribution systems," IEEE Trans. Smart Grid, vol. 6, no. 1, pp. 45-53, 2015.

[5] S. A. Gabriel, A. J. Conejo, J. D. Fuller, B. F. Hobbs, and C. Ruiz, Complementarity modeling in energy markets. New York: Springer, 2012.

[6] R. Palma-Behnke, J. L. Cerda A., L. S. Vargas, and A. Jofré, "A distribution company energy acquisition market model with integration of distributed generation and load curtailment options," IEEE Trans. Power Syst., vol. 20, no. 4, pp. 1718-1727, 2005.

[7] $\mathrm{H}$. Li, Y. Li, and Z. Li, "A multiperiod energy acquisition model for a distribution company with distributed generation and interruptible load," IEEE Trans. Power Syst., vol. 22, no. 2, pp. 588-596, 2007.

[8] J. Lopez-Lezama, A. Padilha-Feltrin, J. Contreras, and J. Munoz, "Optimal contract pricing of distributed generation in distribution networks," IEEE Trans. Power Syst., vol. 26, no. 1, pp. 128-136, Feb 2011.

[9] C. Zhang, Q. Wang, J. Wang, P. Pinson, J. M. Morales, and J. Østergaard, "Real-time procurement strategies of a proactive distribution company with aggregator-based demand response," IEEE Trans. Smart Grid, pp. $1-10,2016$, in press.
[10] C. Zhang, Market design and strategy making for proactive distribution grid with DERs. Denmark: Technical University of Denmark, 2015.

[11] J. Wang, M. Shahidehpour, Z. Li, and A. Botterud, "Strategic generation capacity expansion planning with incomplete information," IEEE Trans. Power Syst., vol. 24, no. 2, pp. 1002-1010, 2009.

[12] C. Ruiz, A. J. Conejo, and Y. Smeers, "Equilibria in an oligopolistic electricity pool with stepwise offer curves," IEEE Trans. Power Syst., vol. 27, no. 2, pp. 752-761, 2012.

[13] R. Fernández-Blanco, J. M. Arroyo, and N. Alguacil, "Networkconstrained day-ahead auction for consumer payment minimization," IEEE Trans. Power Syst., vol. 99, no. 2, pp. 1-11, 2013.

[14] S. J. Kazempour, A. J. Conejo, and C. Ruiz, "Strategic bidding for a large consumer," IEEE Trans. Power Syst., vol. 30, no. 2, pp. 848-856, 2015.

[15] W. Tushar, C. Yuen, D. B. Smith, and H. V. Poor, "Price discrimination for energy trading in smart grid: a game theoretic approach," IEEE Trans. Smart Grid, pp. 1-12, 2016.

[16] G. Liu, Y. Xu, and K. Tomsovic, "Bidding strategy for microgrid in dayAhead market based on hybrid stochastic/robust optimization," vol. 7, no. 1 , pp. 227-237, 2016

[17] M. H. Cintuglu, H. Martin, and O. A. Mohammed, "Real-time implementation of multiagent-based game theory reverse auction model for microgrid market operation," IEEE Trans. Smart Grid, vol. 6, no. 2, pp. 1064-1072, 2015.

[18] A. Soroudi, P. Siano, and A. Keane, "Optimal DR and ESS scheduling for distribution losses payments minimization under electricity price uncertainty," IEEE Trans. Smart Grid, vol. 7, no. 1, pp. 261-272, 2016.

[19] E. G. Kardakos, C. K. Simoglou, and A. G. Bakirtzis, "Optimal offering strategy of a virtual power plant: a stochastic bi-level approach," IEEE Trans. Smart Grid, vol. 7, no. 2, pp. 794-806, 2016.

[20] A. Saint-Pierre and P. Mancarella, "Active distribution system management: a dual-horizon scheduling framework for DSO/TSO interface under uncertainty," IEEE Trans. Smart Grid, pp. 1-12, 2016.

[21] D. Steen, L. A. Tuan, and O. Carlson, "Effects of network tariffs on residential distribution systems and price-responsive customers under hourly electricity pricing," IEEE Trans. Smart Grid, vol. 7, no. 2, pp. 617-626, 2016

[22] M. Z. Degefa, A. Alahäivälä, O. Kilkki, M. Humayun, I. Seilonen, V. Vyatkin, and M. Lehtonen, "MAS-based modeling of active distribution network: the simulation of emerging behaviors," pp. 1-9, 2016.

[23] J. M. Arroyo, "Bilevel programming applied to power system vulnerability analysis under multiple contingencies," IET Gener. Transm. Distrib., vol. 4 , no. 2 , pp. 178-190, 2010.

[24] GAMS, [Online]. Available: http://www.gams.com/.

[25] M. E. Baran and F. F. Wu, "Network reconfiguration in distribution systems for loss reduction and load balancing," IEEE Trans. Power Del., vol. 4, no. 2, pp. 1401-1407, 1989.

[26] D. Zhang, Z. Fu, and L. Zhang, "An improved TS algorithm for lossminimum reconfiguration in large-scale distribution systems," Elect. Power Syst. Res., vol. 77, no. 5-6, pp. 685-694, 2007.

[27] A. Sharma, D. Srinivasan, and A. Trivedi, "A decentralized multiagent system approach for service restoration using DG islanding," IEEE Trans. Smart Grid, vol. 6, no. 6, pp. 2784-2793, 2015.

[28] Nordpool, [Online]. Available: http://www.nordpoolspot.com/.

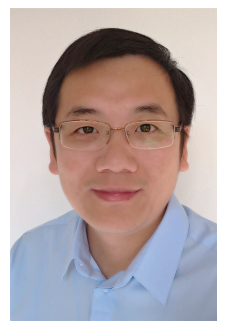

Chunyu Zhang (M'12) received the Ph.D. degree in electrical engineering from Technical University of Denmark (DTU), Denmark, in 2016. From 2006 to 2012, he joined National Power Planning Center and CLP Group as a senior engineer.

$\mathrm{He}$ is currently a postdoctoral fellow at the Department of Electric Power Engineering, Norwegian University of Science and Technology (NTNU), Norway. His research interests include power systems planning and economics, smart grid and electricity markets. 


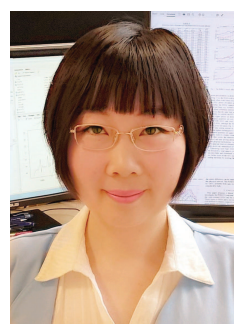

Qi Wang (M'13) received the Ph.D. degree from Technical University of Denmark (DTU), Kgs. Lyngby, Denmark, in 2016, in electrical engineering. She joined East China Electric Power Design Institute as a senior engineer till 2013.

She is currently with Nordea, Filial of Nordea Bank AB, (Publ.), Copenhagen, Denmark. Her research interests are in the fields of economics, planning, markets, risk management, stochastic programming, robust optimization, and complementarity modeling.

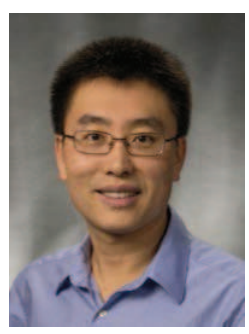

Jianhui Wang (M'07-SM'12) received the Ph.D. degree in electrical engineering from Illinois Institute of Technology, Chicago, IL, USA, in 2007. Presently, he is the Section Lead for Advanced Power Grid Modeling at the Energy Systems Division at Argonne National Laboratory, Argonne, IL, USA.

Dr. Wang is the secretary of the IEEE Power \& Energy Society (PES) Power System Operations Committee. He is an associate editor of Journal of Energy Engineering and an editorial board member of Applied Energy. He is also an affiliate professor at Auburn University and an adjunct professor at University of Notre Dame. He has held visiting positions in Europe, Australia and Hong Kong including a VELUX Visiting Professorship at the Technical University of Denmark (DTU). Dr. Wang is the Editor-in-Chief of the IEEE Transactions on Smart Grid and an IEEE PES Distinguished Lecturer. He is also the recipient of the IEEE PES Power System Operation Committee Prize Paper Award in 2015.

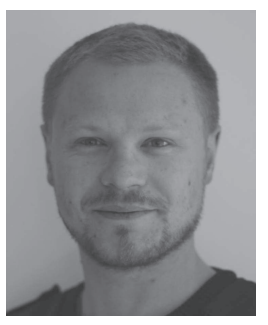

Pierre Pinson (M'11-SM'13) received the M.Sc degree in applied mathematics from the National Institute for Applied Sciences (INSA Toulouse, France) and the Ph.D. degree in energetics from Ecole des Mines de Paris (France). He is a Professor at the Technical University of Denmark, Centre for Electric Power and Energy, Department of Electrical Engineering, also heading a group focusing on Energy Analytics \& Markets. His research interests include among others forecasting, uncertainty estimation, optimization under uncertainty, decision sciences, and renewable energies.

Prof. Pinson acts as an Editor for the International Journal of Forecasting, and for Wind Energy.

Jacob Østergaard (M'95-SM'09) received the M.Sc. degree in electrical engineering from the Technical University of Denmark (DTU), Lyngby, Denmark (DTU), in 1995. He is a Professor and Head of the Centre for Electric Power and Energy, Department of Electrical Engineering at DTU. From 1995 to 2005, he worked for the Research Institute for the Danish Electric Utilities.

Prof. Østergaard is serving in several professional organizations including the EU SmartGrids advisory council. His research interests include system integration of wind power, control architecture for future power systems, and demand side. 